\title{
Application of Data Mining Technology in Network Marketing
}

\author{
Li Kaimin \\ Networking Academy, HaiKou College of Economics, HaiKou 571127, China
}

Keywords: network marketing; digital mining; market management

\begin{abstract}
Nowadays, science and technology in the unceasing progress, enterprises in the process of the development, and various technology applied to marketing and to the development of enterprises have brought some benefits. Although information technology brings some benefits to enterprise management, it will also bring some problems to the development of enterprise. The accumulation of a large amount of data, is the main problem for the enterprises to face, coupled with the more expensive money and effort on data processing from enterprises every year, which has a negative impact on the development of enterprises. The application of data mining technology in network marketing can help enterprises solve these problems effectively, which can not only integrate the data more comprehensively, but also extract more effective information for enterprises. This paper is a detailed description of the specific application of data mining technology in network marketing.
\end{abstract}

\section{Introduction}

The so-called network marketing refers to the Internet as the core network platform, to the user as the center, take the market demand and cognition oriented, to achieve a series of acts of corporate marketing purposes using a variety of network applications. Network marketing has two characteristics, the first is based on the Internet, the Internet as a marketing medium; in addition, it belongs to a marketing category, is the expression form of marketing. Now the network marketing has become increasingly concerned by people, the application of data mining technology to the network marketing, for some companies, not only reduces the cost, but also brings more customers for the enterprise, for the development of enterprises, has a positive effect [1].

\section{Digital Mining Technology}

The so-called digital data mining technology is a kind of data processing technology, is from large, incomplete, noisy, fuzzy and random data, extract the implicit, people do not know, but for the people, but also has some information and process knowledge. According to the need of data mining in data warehouse information, to choose the suitable tool for analysis, and the application of statistical methods, case reasoning, decision tree, rule reasoning, fuzzy sets, neural network, genetic algorithm and other methods of data processing, in order to get the useful information analysis. The digital mining process is a recurring process in which each step is returned to the previous step if each step fails to achieve its intended goal. In the process of data mining, must experience the definition of business, design data model of data warehouse, analysis of mining information, establish data marts, establish model, assessment, the five stages of interpretation and application of the model.

Data mining technology in the actual work, will inevitably be a series of data processing, so as to achieve the desired, the results of data processing. The workflow of digital mining technology mainly includes 8 steps: information collection, data integration, data specification, data cleaning, data transformation, data mining implementation, model evaluation and knowledge representation. The so-called information collection means that the data analysis object samples out the characteristic information needed in the data analysis to determine. Then, the appropriate way to achieve the collection of data information, and then deposited into the database. In the process of mass information processing, it is a very important step to select a suitable data storage and 
management data warehouse. Data integration is to logically and physically collect data from different sources, different formats, different characteristics, and different properties, so as to provide comprehensive data sharing for enterprises. Data specification refers to the implementation of the majority of data mining algorithms, even in a small amount of data also takes a long time, and in the process of commercial operation, the amount of data mining is often larger. Data reduction techniques can be used to obtain the data set protocol said that this data set is small, but still be able to close to maintain the integrity of the original data, and the protocols and protocol after the implementation of data mining results before the results are almost the same. Data cleaning refers to the fact that in the database there are always incomplete, noisy, and inconsistent data. Therefore, the data need to be cleaned up, and the complete, correct and consistent data information will be stored in the data warehouse, otherwise the result of data mining will be unsatisfactory. The data transformation refers to the smooth data aggregation, generalization, standardization, data will be converted into a form suitable for data mining for real data, based on the discrete data and the concept of hierarchical data conversion is a very important step. The data mining model is based on the data in the data warehouse information, select the appropriate analytical tools, and using statistical methods and case reasoning, decision tree method, rule reasoning, fuzzy sets, neural network and genetic algorithm to the processing of information, to obtain useful information analysis. Schema evaluation is the validation of data mining results from a business perspective. Knowledge representation refers to the analysis information obtained by data mining to the user in a visual way, or stores it in the knowledge base for other applications [2].

\section{The Main Application of Digital Mining Technology in Network Marketing}

Network marketing has become the enterprise now is one of the main ways of marketing, digital mining technology applied to the network marketing, for business development, also has some good effects, the following is the specific application of data mining technology in network marketing in detail.

For the development of an enterprise, customer relationship management has a vital role to the enterprise, to establish a close relationship between how to retain customers, and customers, mining potential customers, the customer demand analysis, meet customer requirements, for the development of enterprises, is a very important marketing the problem. The so-called customer relationship management, refers to the enterprise used to manage customer relations tool. Customer Relationship Management (CRM) is a continuous process that constantly strengthens customer interaction, keeps abreast of customer needs, and continuously improves and improves products and services to meet customer needs. The new management concept emerged in the late $90 \mathrm{~s}$. The main purpose of customer relationship management, is to realize the interaction between with customers, so as to effectively enhance the customer satisfaction, customer loyalty can be effectively improved, and customer relationship management to carry out mining of potential customers, so as to improve the enterprise and the customer relations, to meet customer needs, promote social and economic development objective. Data mining technology can be applied to many different stages and customer relationship management, including: first, let business people understand the importance of customer, the customer is not the key of survival and development of trade, but in the field of enterprises, but also to let customers have a certain understanding of business processes. Two, to ensure that enterprises and users maintain a sustainable development relationship, this way can make customers and enterprises better exchanges between each other, and make more profits. Three, the profitability of the customer can be analyzed, because the customer profitability is different, only the customer profitability reasonable analysis, in order to develop more effective marketing strategies, so as to obtain the most valuable customer. In addition, also can effectively improve customer loyalty, and according to the analysis of the data to find the most close to the customer purchase behavior, so as to improve the transaction with the customer profitability, and to develop new customers. Four, now departments are becoming increasingly involved in maintaining customer relationships, and businesses need to increase costs to get more new customers. Therefore, for enterprises, maintaining the relationship with the original customer becomes more important. 
Now enterprises in the development process, the customer can be divided into three types, the first is the value of low or no value to the user; and is not easy to lose valuable customers, such customers for marketing activities, has a certain potential. Finally, more valuable customers, for such customers, tend to bring greater value to the enterprise, but such customers need enterprises to ensure their own operating costs, but also to tap. Five, customers in the enterprise business resources system access process, we must classify different customers, in order to better ensure the development of enterprises [3].

In the credit assessment and risk analysis, data mining technology has been used in some enterprises in the process of marketing, there will inevitably be a buyer's credit risk problem, and now the market competition is so fierce, customers in the selection process of enterprise, trade credit has become an important condition. And customer credit management can collect the information of customers, so that they have a certain understanding of the biggest source of wealth after the enterprise, and also have a certain grasp of the risk. The application of data mining technology to the network marketing, can carry on the reasonable analysis of the data, so as to avoid some of the fraud and deferred payment behavior, therefore a detailed grasp of customer information, making decision is very important for enterprise security. In a word, the digital mining technique applied to credit risk management, has the following advantages: first, it can automatically form a simple evaluation model, and for any number of data are enough for statistical and inductive reasoning, and can easily get the conclusion that the data evaluation of this way is effectively reduced in the actual application of the cost. Two, for enterprise financial standards and credit problems, for enterprises, the use of data mining technology is more suitable for describing it. In the process of data processing, the nonlinear characteristics are all online, and the data is analyzed in an appropriate way. In the enterprise's credit status and financial indicators, the realized are non-linear features, which makes data mining technology can show its own role. Three. Data mining techniques are capable of processing various types of data, whether continuous, discrete, or otherwise. Four, the data mining technology to noise data are corrected, now the face of such a market, there are always false data and information, data mining can be these error information is correct, so the accuracy of the model has been improved to some extent. Five, data mining technology can calculate incomplete information, and optimize the range of performance through classification tree and rough set data analysis method, so as to obtain more accurate index estimation value [4].

\section{The Advantages of Data Mining in Network Marketing}

With the continuous progress of society, the form of economic development has gradually developed to the virtual operation of the network, and network marketing has gradually become a new marketing theory which is more adapted to the current market. Network marketing can select the most valuable information from many data for enterprise staff. And data mining technology for data processing is further, able to deal with past data, and thus find the potential link, which is more important for enterprise personnel.

The application of data mining technology to network marketing can maintain the existing customers, and also tap some potential customers. Because, in the network marketing sales staff for customer information will be retained through a query to access records, to understand some of the potential customer information, according to the information, and then make some targeted recommendations, to attract customers, so take, will retain customers. Moreover, data mining techniques can mine web logs, classify customers in detail, and find potential new customers [5].

The application of data mining technology in network marketing, managers can grasp the true information of this technique is the feedback of the market, so that we can timely understand customer needs for future products, according to customer preferences, adopt appropriate marketing solutions. In addition, the customer can access used for data analysis, in place of product advertising, so that you can make some advertising to promote, this way can effectively improve the reporting rate of advertising, so as to achieve the purpose of reducing operating costs [6].

The application of data mining technology in network marketing, you can store the data and 
processing transactions for customers, can think of feedback control, based on that information, managers can design the network marketing website. After the designer optimizes the website according to the information, may give the user to bring the more convenient browsing way.

Applying data mining technology to network marketing can make personalized recommendation to customers. The so-called "personalized" service is to face different customers, different service strategies and service content, that is, to achieve customer centric web services needs. After collecting and arranging the customer's information, we will be more informed about the customer's purchasing behavior, and then take the initiative to achieve the suggested service.

\section{Conclusion}

Today, the social development is so fast, science and technology also gradually into people's lives, people are more and more dependent on online shopping, and network marketing data mining has become a more promising field. In network marketing, application of data mining technology, capable of data effectively, get useful information, choose the marketing plan of benefit, retain old customers at the same time, also to carry out mining of potential customers, the enterprise's development, has the vital significance. Not only that, data mining technology has also solved many problems for enterprises, and brought huge profits for enterprises. In a word, the application of data mining technology in network marketing, has a huge advantage, also hope that through this article, the data mining technology can get more extensive application, to better promote the development of the national economy.

\section{References}

[1] Lu Z. Research on the Application of Data Mining Technology in Network Marketing[J]. Electronic Technology, 2012.

[2] Ma X L, Zhang X L. The Application of Data Mining Technology in Computer Network Security[C]// Seventh International Conference on Measuring Technology and Mechatronics Automation. IEEE, 2015:1126-1129.

[3] $\mathrm{Hu} \mathrm{J} \mathrm{F,} \mathrm{Li} \mathrm{B} \mathrm{S.} \mathrm{Research} \mathrm{on} \mathrm{the} \mathrm{application} \mathrm{of} \mathrm{web} \mathrm{data} \mathrm{mining} \mathrm{technology} \mathrm{in} \mathrm{tourism}$ E-Commerce[C]// World Automation Congress. IEEE, 2012:1-4.

[4] Shen Z H, Peng W P. Research of E-business Application based on Data Mining[C]// 2008 international conference of management science and engineering. 2008.

[5] Zhang Z, Chang G R, Huang X Y. The Application of Data Mining Technology in CRM[J]. Chinese Journal of Management Science, 2003.

[6] Shin H M, Jeong D H. An application of data mining for marketing in telecommunication[C]// Portland International Conference on Management of Engineering and Technology. IEEE, 2001:247 vol.1.

Kaimin Li Female, Hainan province, in may 1982. undergraduate, A lecturer, Network technology and computer application. 